\title{
Time perception, immersion and music in videogames
}

\author{
Timothy Sanders \\ Stevenson CollegeCRAFT \\ Edinburgh \\ tsanders@stevenson.ac.uk
}

\author{
Paul Cairns \\ Dept. of Computer Science \\ University of York York YO10 5DD, UK \\ pcairns@cs.york.ac.uk
}

\begin{abstract}
People who play videogames often report the sense of immersion in the game with a particular feature of immersion being a loss of the sense of time passing. In this paper, we investigate if altering the degree of immersion in a videogame really does influence people's psychological perception of time passing. We use music to make a maze game more immersive and we measure time perception using two paradigms that are well-established in psychology. We find that the addition of music does alter time perception but only in one paradigm. Additionally, music was able to influence immersion by both increasing it or decreasing it depending on the choice of music. The overall picture is therefore complex but suggests that music could be an important factor in the perception of time whilst playing videogames.
\end{abstract}

time perception, immersion, videogames, music, gaming experience

\section{INTRODUCTION}

The experience of immersion in videogames is commonly reported by players, reviewers and designers of games [5]. One component of immersion that is dominant is that players report losing their sense of time passing and indeed this can be viewed as both a good and bad aspect of the overall experience of playing videogames [24]. This paper therefore sets out to use estimates of specific durations to see how the experiential report of immersion relates to an actual variation in the perception of time. We describe two experiments where participants played a maze game and the degree of immersion was manipulated by adding music. Participants were then asked to report on the estimated duration of play, both with and without prior knowledge that this question was coming. The results suggest that players genuinely do lose a sense of time when more immersed but that the addition of music seemed to have its own effect on players. The results also suggest that the ways in which players estimate time varies depending on whether they know they are going to have to produce a time estimate. The work however does not provide the (hoped for) definitive answers but does suggest that time perception whilst playing videogames is a rich and complex phenomenon.

\section{IMMERSION AND TIME PERCEPTION}

Whilst the notion of immersion is reported by gamers and can be reliably measured,eg [14], in the academic literature the term immersion has multiple meanings and it is worth being clear first what we mean before turning to current understandings of the perception of time. This then allows us to set up the context for manipulating immersion in order to investigate time perception.

\subsection{Immersion}

Immersion as understood here is the sense of being "in a game" where a person's thoughts, attention and goals are all focused in and around the game as opposed to attending to being concerned with anything else, such as what is going on in the room around them. Immersion must first be distinguished from the notion of presence [19]. Presence is associated with virtual reality environments and is the sense a person has of actually being located in the virtual space. Virtual reality environments are used in many first person shooter games, such as Rogue Trooper, but there are also specialist systems such as CAVEs that provide a room-sized display that surrounds a person. Such virtual reality systems are described as immersive in the sense that a person can feel as if they have entered the environment. However immersion in this sense is a property of the system that allows a person to feel presence. It is clear though that it is possible to feel present in a virtual environment but not immersed in what you are doing there, for example doing some boring task. By contrast, there are many games, such as Tetris, where there is no virtual world for a player to feel present in but that do definitely provide an immersive experience. Another concept similar 
to immersion is that of flow [8]. Flow however is an extreme experience where goals, challenge and skill converge. As such flow is an all or nothing experience. By contrast, immersion in games is a much more prosaic experience and graded with low and high levels of immersion possible. Gamers recognise that the intensely immersive experiences that might be regarded as flow are at best fleeting or transitory [5]. It should also be noted that flow has been considered in the context of videogames through the notion of GameFlow [20]. However, as defined, GameFlow is a property of games and not an experience had by players and thus does not address notions of immersion and involvement addressed here.

Thus overall, immersion in games is a cognitive experience constructed within the mind of a player. It does not need to be an extreme experience and whilst about being "in a game" this is not meant in any physical or transported sense but rather about a players thoughts and feelings being more involved in the game than anything else.

\subsection{Time perception}

Timing and a sense of time, be it future, present or past, is an essential part of human actions and behaviour. Fundamental human activities like talking and walking require well-timed sequences of muscle actions. On the larger scale, many aspects of life also require a good understanding of time and the passage of time in order that we can make plans, coordinate activities or even just show up for work! Accordingly, time perception has been an important part of psychology from its earliest days, for example, James' seminal text of 1890 [12].

However, unlike other aspects of perception, there is no physical manifestation of time in the same way that there are objects to see and touch, sound waves to hear and so on. Nor is there any process internal to ourselves that corresponds to a timepiece. Instead, many theorists explain duration experiences in terms of cognitive processes or interactions between cognitive and biological processes [3].

What has been clear from the outset of studying time perception is that people perceive time differently depending on how they think (or do not think) about time. These are captured in the common idioms of "A watched pot never boils" and "Time flies when you are having fun." Studies by James [12] made the distinction between different types of duration experiences. He stated that different variables affect a person's prospective and retrospective sense of time. The perception of elapsed time can therefore be measured under two paradigms. In the case of a prospective paradigm, a person is aware that they needed to make a time estimate before experiencing a duration. The prospective paradigm is also known as experienced duration. In the case of a retrospective paradigm, a person is unaware of the need for a time estimate until after the time period has passed. The retrospective paradigm is also referred to as remembered duration [3].

Though there is some belief that the two types of duration judgments use similar cognitive processes [7], there has emerged an increasingly dominant view that this is not the case [2, 10, 25, 26]. In order to try to come up with a clear view on the differences between retrospective and prospective time estimation processes, Block and Zakay conducted a meta-review of 20 experiments [3]. They found that there was one variable that affected prospective time estimates but not retrospective ones, this being the processing complexity of an experimental task, for example, a simple vs a complex Stroop task [26]. As the processing complexity of the task increases, prospective time estimates decrease suggesting that participants have less attention to allocate to the monitoring of time. This of course is not an issue in the retrospective condition.

Conversely, the length of the duration to be estimated and the stimulus complexity, for example being presented more complex visual patterns [1], both affect retrospective time estimates but not prospective ones. Retrospective estimates must be based on some form of memory and as stimulus complexity increases, this provides more memories for retrospective judgments to attach to. It is not clear why increasing length of stimulus duration affects the time estimation.

Taken together, the different effects of different variables across prospective and retrospective paradigms suggest that the two time estimation methods use distinct cognitive processes. The current theory is that for prospective time estimation attention is needed to monitor time passing. In some sense, a person is mentally counting the ticking of some internal clock. However, the more a person's attention is required elsewhere, the more ticks they miss and hence underestimate time. For retrospective time estimates, a person looks back over their memory of a duration and essentially counts the memories. The fewer contextual changes requiring distinct memories, the lower the time estimate.

One consistent finding across both paradigms is that if players are given two tasks to do, one temporal, one not, then there is a strong interference effect with the secondary task causing the time estimations to become shorter, more variable or more inaccurate [6].

\subsection{Manipulating immersion for time perception}

Given the complexity of time estimation processes, it is something of a challenge to find out exactly what is happening to players' perceptions of time as they 
play videogames. There have been a few attempts to actually measure players' time estimates of how long they have been playing. Wood et al [24] found in a survey of gamers that time loss was commonly reported by gamers and that it had both positive and negative connotations. Tobin et al. [21] studied players time estimates in an ecologically appropriate environment where players were playing for many minutes (up to 58) and being asked to produce either retrospective or prospective estimates of playing duration. Surprisingly, it was found that though there was a difference between paradigms, players did not at all underestimate their duration of play. Rau et al. [18] also used long durations of play, 30 mins or 60 mins, and ran their study in a CyberCafe environment. They were looking at the difference in time estimation between novice and expert players. They also found that players tended to overestimate playing durations and even the expert players who played for an hour only made a marginal underestimate (of around 2 minutes on average) which can be inferred from the reported results to be not significantly different from the correct value of 60 mins. It is not clear whether a retrospective or prospective paradigm was used.

It is possible that the very ecological validity in both of these studies undermined players' time estimates. They could have picked up cues from their environment as to elapsed time, for example, changing light conditions or the coming and going of other people. Alternatively, over long periods people may have compensation mechanisms that allow them to revise their psychological experiences. That is, whilst they may have actually had a distortion in their perception of time, they are able to reflect on their experience, perhaps by using cues from their own physiological state such as being thirsty or uncomfortable, so that they can correct for what they felt as elapsed time to produce reasonably accurate time estimates.

Luthman et al. [16] used a totally different paradigm to look at enduring time distortion after the period of play, using a production method rather than verbal reports to get players to produce a given time interval. They also worked in an ecologically valid environment, namely a LAN party where players get together to play games usually over a weekend. Again what is noticeable is that after playing, players tended to produce more accurate time intervals than in the control condition where they had not played beforehand.

There have been attempts to measure the change in time perception in relation to presence. Where there was no task for people to do, the sense of presence seemed to bear no relation to their sensation of time passing [22]. Hagni et al. [9] did provide participants with different tasks that were intended to manipulate presence. However, presence was only probed simplistically using a single question and, moreover, that question asked about how immersive the task was and not about the sense of presence. The findings suggested that increased immersion was altering players' ability to reproduce a given duration whilst doing a task but this could simply be due to increasing complexity of the tasks in the experimental set-up.

So overall, whilst time loss is a reported experience of playing games, studies seem to suggest that players are in fact quite able to maintain an accurate sense of time passing. What is noticeable though in the previous work is that there was neither any attempt to manipulate the games being played nor any efforts to measure the degree of engagement of players. We therefore chose to move to a lab setting to provide much greater control over what players were experiencing and also reducing the time of play substantially to a matter of minutes. Previous research has shown that this has still been sufficient to get differing levels of immersion [13] but it makes it unlikely that other physiological changes will take place within the player. Additionally, we measured immersion to see that the different settings really were providing a different experience to players.

Nonetheless, for measuring the association between time perception and immersion, there remains the challenge of devising a playing situation where immersion can be manipulated but other aspects known to influence time perception are not. For a specific game, any manipulation of the game that alters the gameplay could affect either processing complexity or stimulus complexity both known to alter time estimates. Thus, immersion cannot be manipulated by altering the game. Furthermore, if players are given some secondary task to do external to the game, there is bound to be interference which will affect time perception.

To attempt to solve this problem, we decided on the addition of music as a mechanism for manipulation of immersion. In previous studies (currently in preparation), we have found that adding music could substantially increase the immersive experience of playing a game. Also, it is well recognised that music is an integral part to adding to the feel and experience of playing a game [23]. Music however is not a necessary part of the game and so, in terms of the stimulus or processing complexity of the task in hand, the game itself does not change. Nor does the music add a secondary task to the game as there is no requirement to attend to the music in any way. Music has also been shown to alter time perception [15] but tending to cause overestimation of duration in otherwise empty, waiting intervals. Thus if music is having an effect independent of immersion, we should expect to see time gain in the time estimates not time loss. 


\section{EXPERIMENT 1}

The main goal in this experiment is to investigate the relationship between levels of immersion and time estimates by manipulating the immersiveness of a game using music. The control group therefore plays a game without music and the experimental group has the same game but with a music soundtrack added. The immersion levels of the participants were measured by a questionnaire [14], while time estimation was measured by a participant estimated written scale with both retrospective and prospective paradigms. It is believed that a change in immersion levels will alter players' ability to estimate time. Therefore, the hypotheses are:

1. Immersion will be higher in the experimental group

2. Prospective and retrospective time estimates in the experimental group would be significantly different than those in the control group.

\subsection{Participants}

Forty one participants were recruited, 36 of whom were from academia (e.g. student, research assistant, teacher or lecturer). One participant's data was omitted due to interruptions in the testing conditions. Of the 40 participants, 21 were women and 19 were men. They had an age range of 21 to 55 years of age. All participants had experience with video or computer games before. The average number of years of playing computer games on a regular basis was just less than 3 to 5 years (just over 1 to 3 for females and 3 to 5 years for males). On average, participants play videos games more than 2 to 3 times a week for an average duration of just under 1 to 3 hours.

\subsection{Design}

The experiment was a two-way factorial, between subjects design. The two factors are protocol, retrospective and prospective, and immersion, with music and without. Each participant was allocated randomly to one of the four conditions but balanced to give ten participants in each condition.

\subsection{Materials}

The experiment was conducted using a Lenovo widescreen laptop. The game was a $3 \mathrm{~d}$, first person maze game ${ }^{1}$.The music for the experimental condition was taken from The Legend of Zelda: Ocarina of Time, specifically the music for a "boss battle." It is a fast paced piece but also quite jarring and discordant. The music and game were played

${ }^{1}$ Available at: www.falstad.com/maze within the Google Chrome browser that supported Java used in full screen mode for a seamless interface. The Windows interface was also closed to prevent accidental use of it. The participants in the experimental group also used Sony studio headphones to listen to the music. This was used to make sure that room acoustics and external noise do not interfere with participants while playing. The room used was well lit and had the curtains drawn. It was made certain that participants could not see any timepiece during the running of the experiment.

Table 1: Means (and standard deviations) of immersion scores in the four conditions in experiment 1

\begin{tabular}{l|r|r} 
& \multicolumn{2}{|c}{ Protocol } \\
& Prospective & Retrospective \\
\hline With Music & 131.7 & 124.4 \\
& $(23.2)$ & $(12.9)$ \\
\hline Without Music & 138.5 & 144.6 \\
& $(23.6)$ & $(13.4)$
\end{tabular}

Demographic details were gathered using an initial questionnaire covering factual matters such as age, gender, occupation and participants gaming history including frequency of play, average playing duration and the amount of years they have been playing. Immersion was measured using an immersion questionnaire with 31 questions answered on 7 point Likert scales[14]. An extra question was asked at the start of the questionnaire asking players to estimate the duration of playing time. Initially this last question was simply an open answer however it was found that players struggled to generate sufficiently precise estimates without substantial prompting. Thus, we used a closed choice question where timing estimates were given in 30 second intervals, starting with 60s-90s and going up to more than 5 mins. Participants had to choose the interval they thought most likely [17]. Further questions were added to the end of the immersion questionnaire to probe how much people liked playing this game and this sort of game.

\subsection{Procedure}

Having discussed and received informed consent, participants were introduced to the game and its controls and given a chance to try them out. For the experimental group, the headphones were adjusted to fit the participant and the volume was adjusted to their liking. Participants in the prospective group were then instructed to keep track of time while playing. All participants were also told to act as if no one was in the room while they were playing. Once we were confident that the participant understood the task and instructions, they were instructed to play until they were told to stop. The game then started. Playing duration lasted for 3 minutes and 23 seconds (203 seconds). Participants at this time 
were told to stop playing and instructed immediately to estimate the duration of play and then complete the immersion and demographic questionnaires.

\subsection{Results and Discussion}

Immersion was scored according to [14] to give a score with a possible range of 31 to 217 . The immersion scores are summarised in Table 1. It is immediately apparent that immersion in the experimental condition, that is with music, is actually lower than in the control condition. This difference is significant, $F(1,36)=5.06, p=0.031$ but there is no difference in immersion between the protocols, $F(1,36)=0.01, p=0.921$ or any interaction effect, $F(1,36)=1.25, p=0.272$.

The time estimate intervals were scored from 1, being the shortest interval of 60-90s, up to 11 being 5 or more minutes. The correct interval for the gameplay duration was 6 though 7 would also be a reasonably close estimate. The time estimates are summarised in Table 2.

Table 2: Means (and standard deviations) of interval scores in the four conditions in experiment 1

\begin{tabular}{l|r|r} 
& \multicolumn{2}{|c}{ Protocol } \\
& Prospective & Retrospective \\
\hline With Music & 5.2 & 5.3 \\
& $(2.7)$ & $(1.5)$ \\
\hline Without Music & 7.3 & 4.8 \\
& $(1.8)$ & $(1.5)$
\end{tabular}

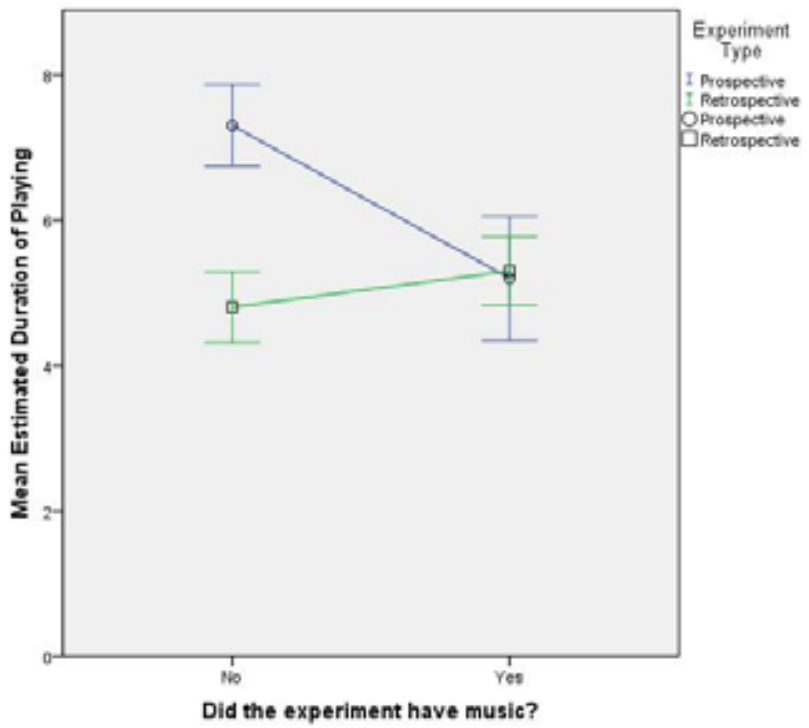

Figure 1: Mean estimated duration in interval scores \pm 1 standard error in the four conditions of experiment 1

There is no significant difference between time estimates for the experimental and control groups, $F(1,36)=1.72, p=0.20$, nor between protocols $F(1,36)$ $=3.83, p=0.058$, though this latter is approaching significance. There is a significant interaction effect $F(1,36)=4.49, p<0.041$. The interpretation is that, on average, the prospective, control condition stands out from the others with correct time estimates whereas all three other conditions underestimate the elapsed duration of play by one interval, or around $30 \mathrm{~s}$. This can be seen more clearly in Figure 1 where the means of each condition are plotted with one standard error.

These findings do not support our intended aim of increasing immersion in order to see the effect on time perception. Previous studies have suggested that the addition of music could increase immersion however, we have also found that how much a person likes a game can dominate their immersion over and above other factors (papers in preparation). With this in mind, the three liking questions that specifically probed on liking playing this game were summed to give a Likability score. These are summarised in Table 3. As expected, this shows that the Likability score is lower in the experimental condition and indeed this difference is significant, $F(1,36)=$ $6.37, p=0.016$ and there is no effect for protocol, $F(1,36)=0.52, p=0.475$, or interaction effect, $F(1,36)$ $=0.98, p=0.328$.

Table 3: Means (and standard deviations) of Likability scores in the four conditions in experiment 1

\begin{tabular}{l|r|r} 
& \multicolumn{2}{|c}{ Protocol } \\
& Prospective & Retrospective \\
\hline With Music & 10.2 & 9.9 \\
& $(3.9)$ & $(3.7)$ \\
\hline Without Music & 11.9 & 13.8 \\
& $(3.3)$ & $(2.9)$
\end{tabular}

Thus overall it seems that the set up failed to induce immersion because the participants disliked the music used. It is still notable though that the prospective condition in the control group produced generally more accurate predictions than any of the other conditions. This suggests two things, first that the retrospective protocol does cause people to underestimate time of play for this game and that the addition of music, albeit disliked music, has an effect on prospective time perception but not on retrospective.

\section{EXPERIMENT 2}

The goal of this experiment was to overcome the problem with the music that was not liked in the first experiment and thereby produce an increase in immersion in the experimental group. To avoid the problems encountered with the previous music, a short survey was made with ten people to see which they liked most out of four candidate pieces of music. They were asked to rate the music for how thrilling it was, the sense of urgency it suggested, whether it was fun and whether it was enjoyable. Overall, one piece stood out from the others and this piece, part of the sound track to the film The Bourne Identity, was chosen for this experiment. 


\subsection{Method}

The method used in experiment 2 was identical to that of experiment one except for the different piece of music used in the experimental condition.

\subsection{Participants}

There were thirty-three participants in total, eight in each condition and one extra in the retrospective experimental condition. As before the majority of them, 26 out of the 33, were from an academic background. Of these participants, 13 were women and 20 were men. The ages ranged from 22 to 55 . All participants had experience with videogames previously. The average number of number of years of playing video games on a regular basis was slightly greater than 3 to 5 years (just under 3 to 5 years for females and slightly less than 5 to 10 years for males). On average, participants played videogames at least once a week for an average duration of one to three hours.

\subsection{Results and Discussion}

To confirm that the experimental manipulation was having the desired effect of increasing immersion, the immersion scores were compared in each condition. Table 4 summarises the means and standard deviations. As hoped, the immersion score does goes up in the experimental condition and this difference is significant, $F(1,29)=10.47, p=0.003$ and there is no significant differences between protocol, $F(1,29)=1.59, p=0.217$ or an interaction effect, $F(1,29)=0.73, p=0.401$.

Table 4: Means (and standard deviations) of immersion scores in the four conditions of experiment 2

\begin{tabular}{l|r|r} 
& \multicolumn{2}{|c}{ Protocol } \\
& Prospective & Retrospective \\
\hline With Music & 132.8 & 134.7 \\
& $(14.3)$ & $(10.2)$ \\
\hline Without Music & 115.0 & 124.3 \\
& $(11.6)$ & $(14.0)$
\end{tabular}

Table 5: Means (and standard deviations) of interval scores in the four conditions in experiment 2

\begin{tabular}{l|r|r} 
& \multicolumn{2}{|c}{ Protocol } \\
& Prospective & Retrospective \\
\hline With Music & 3.9 & 5.3 \\
& $(1.5)$ & $(1.0)$ \\
\hline Without Music & 6.3 & 5.0 \\
& $(1.9)$ & $(1.6)$
\end{tabular}

Turning to time perception, the time estimations by 30 s intervals are summarised in Table 5 . What is most noticeable in comparison with the results for the previous experiment is that in the experimental condition of the prospective protocol, the time estimation is substantially lower than in either retrospective condition whereas in the control condition it is still quite high and in the correct bracket for the true elapsed playing time. This more complicated picture is reflected in the ANOVA analysis where there is no main effect for protocol, $F(1,29)=0.04, p=0.845$, or experimental condition, $F(1,29)=3.75, p=0.063$ but there is an interaction effect, $F(1,29)=6.59, p=0.016$.

It is difficult to analyse a two-way ANOVA further as there are no agreed upon tests to do this. Figure 2 plots the means in each condition with one standard error. Following Howell [11], Fisher's LSD was used to explore any differences in cell means. This gives that the prospective, experimental condition is significantly different from the prospective control and the retrospective experimental conditions and nothing else. Statistics are not given here as they, at best, give an impression of the interaction effect. Further details are available from the second author on request.

\section{DISCUSSION}

It is easiest to begin by discussing what the two experiments have in common. Most striking is that across both experiments, there is a high degree of consistency in the average retrospective estimates of time and it consistently underestimates the duration of the game play by one time interval, that is $30 \mathrm{~s}$, over the $3 \mathrm{~m} 23 \mathrm{~s}$ of playing. A one-sample t-test was used to explore this difference. It indicates that over both experiments and all groups, the retrospective time estimates average at 5.11 (sd $=1.39$ ) and this is significantly below the correct time interval label which is $6, t(36)=\cdot 3.90, p<0.001$. This underestimation is typical of time estimation experiments [3].

The similarity between experimental and control groups suggests that the addition of music was having no effect on retrospective estimation of time. The theory of durational neglect for retrospective time estimates [4] offers an explanation in terms of the amount of memory needed to remember playing the game. In all cases the cognitive context of the maze game did not vary and hence each version of the game laid down a similar quantity of memories. That is, the musicdid not alter how people remembered the duration. It is also worth noting that whilst music might make the game less boring, this factor has not been found to influence retrospective time estimates [3].

The other common finding in both experiments is that the average prospective estimates in the control condition are correct giving the time labels of 7 or 6 to the duration of playing. This suggests that navigating the maze alone did not require a substantial amount of information processing or attentional resources otherwise the prospective estimates would have been generally less than the actual duration $[3,6]$. 


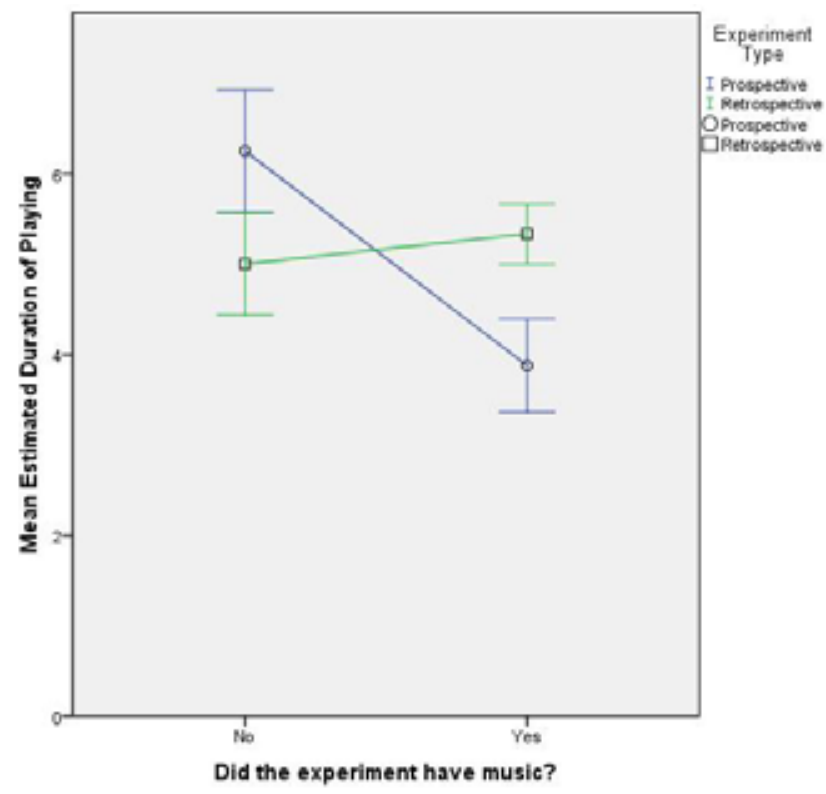

Figure 2: Mean estimated duration in interval scores \pm 1 standard error in the four conditions of experiment 2

Thus, whilst the immersion scores suggest that the game is quite immersive, it is clearly not requiring too much thinking about to be played. This perhaps reflects some experiences of playing where a person is not highly challenged by playing a game that is perhaps familiar or easy for them but nonetheless, they are engaged and it helps to pass the time [24]. Of course, the major difference between the experiments is the effect on prospective timing in the experimental condition. In both experiments the addition of music caused players to underestimate the duration of play. The question then is: what caused this reduction? The game with music is clearly more complex than just the game but adding music does not alter the gameplay in any way - the task is still to navigate the maze and the music simply adds atmostphere. Thus, there is no requirement for increased attention to the game as the result of the addition of music. Nor can the music be increasing stimulus complexity since this alone does not cause alterations in prospective time estimates [3]. In fact, if there were an increase in stimulus complexity, it would have caused an increase in retrospective time estimates. This did not happen either. Thus the addition of music is not adding to stimulus complexity nor is there increased processing complexity of the task. Something else must be happening to affect time estimation.

There is then the added effect that, in Experiment 2 experimental condition, the prospective judgment of duration was significantly lower than the retrospective judgment. This suggests that the addition of likeable music was having some further effect on the players. It is tempting to ascribe this effect to immersion as this did increase in the experimental condition. However, given the complex picture presented by both experiments, we are understandably cautious. If the effect is due to immersion, the explanation would be that players were drawn more into the game by the music and hence, despite the game not requiring more attention, players were not allocating attention to the task of monitoring time.

A more cautious interpretation is that music draws away attentional resources whether relevant to the task in hand or not. The more likeable the music, the more attention it attracts and hence the less a player is able to reliably track the passing of time. In this sense, it is possible that the addition of music sets up one of the conditions for immersion, namely that players become less aware of time and hence promotes the possibility of becoming immersed. That is music, like perhaps dimming the lights and taking the phone of the hook [5], sets up the environment where immersion is a more likely possibility. It is tempting to think that perhaps listening to music simply makes time pass faster for listeners but, as mentioned previously, this is not the case [15] and people who listen to music whilst waiting tend to overestimate the elapsed duration. There must be some integration of the music with the task of playing the game that causes the under-estimation of perceived time.

One unexpected outcome of this work is that the addition of music offers a new variable that can be seen to affect prospective time estimates but not retrospective ones. This further adds to the theory that the two paradigms use different cognitive mechanisms but what is not explained is how music influences the prospective mechanisms.

There is clearly then enormous scope for further work in this area. Different games with a wider range of music types would certainly help to fill out the picture that is beginning to emerge here. Is it that any music at all will alter prospective time estimates? Or are there some sorts of music that have no effect? It is conceivable that the sorts of music that affect players are highly dependent on cultural background. And is there some factor, other than likeability, that means music increases immersion, for instance congruence with the game or "adding atmosphere"? The maze game is in some sense an emotionally neutral game as it does not require any particular emotional perspective but many games rely on building up a mood, such as fear or happiness, that music could tap into or interfere with in some way.

The experimental manipulation itself could be altered so that immersion is increased in some way that does not use music. But as discussed earlier, any alteration of the game-play itself alters the information processing requirement of the game and is therefore known to affect time estimation independently to immersion. What is needed is some way to alter the context of the game without altering the game. It might be possible to achieve this by altering the motivation of players through the use of prizes or cash bonuses for high scores though it is not known if this is a mechanism that does increase immersion. 


\section{CONCLUSIONS}

In summary, music added to a game is able to cause duration to be under-estimated in the prospecive paradigm but not the retrospective paradigm. That is, music reduces the experienced duration of playing a game but not the remembered duration. What is more, the addition of music can make playing more or less immersive depending on whether the music is liked or not. It may even be the case that music is able to set up the situation where immersion is more likely to occur. This is a complex picture and the work described is only just scratching the surface of what might actually be going on with music in videogames. What does seem clear though is that this whole area would benefit from the investment of a lot more time.

\section{ACKNOWLEDGMENTS}

Many thanks to Paul Falstad for allowing us to use his maze game in our experiments and to Anna Cox for her useful comments.

\section{REFERENCES}

[1] R. A. Block. Remembered duration: effects of event and sequence complexity. Memory and Cognition, 6(3):320-326, 1978.

[2] R.A. Block. Prospective and retrospective duration judgment: The role of information processing and memory. In F. Macar, V. Pouthas, and W. J. Friedman, editors, Time, action and cognition: Towards bridging the gap, pages 141-152. Kluwer, Dordrecht, 1992.

[3] R. A. Block and D. Zakay. Prospective and retrospective duration judgments: A meta-analytic review. Psychonomic Bulletin and Review,4:656-665, 1997.

[4] R. A. Block and D. Zakay. Timing and remembering the past, the present and the future. In S. Grondin, editor, Psychology of Time, pages 367-394. Emerald Group, Bingley, UK, 2008.

[5] E. Brown and P. Cairns. A grounded investigation of immersion in games. In ACM CHI 2004,pages 1297-1300, 2004.

[6] S. W. Brown. Time and attention: Review of the literature. In S. Grondin, editor, Psychology of Time, pages 111-138. Emerald Group, Bingley, UK, 2008.

[7] S. W. Brown and D. A. Stubbs. Attention and interference in prospective and retrospective timing. Perception,21:545-557,1992.

[8] M. Csikszentmihalyi. Flow: The Psychology of Optimal Experience. Harper and Row, New York, 1990.

[9] K. Hagni, K. Eng, M.-C. Hepp-Reymond, L. Holper, B. Keisker, E. Siekierka, and D. C. Kiper. The effect of task and ownership on time estimation in virtual environments. In Presence,pages145-150, 2007.
[10] R. E. Hicks, G. W. Miller, and M. Kinsbourne. Prospective and retrospective judgments of time as a function of amount of information processed. The American Journal of Psychology,89(4):719-730, 1976.

[11] D. C. Howell. Fundamental Statistics for the Behavioral Sciences, Sixth Edition.Thomson Wadsworth, Belmont, CA, 2008.

[12] W. James. The principles of psychology. Dover Publications, Mineola, New York, 1957.

[13] C. Jennett, A. Cox, and P. Cairns. Investigating computer game immersion and the component real world dissociation. In ACM CHI 2009,pages 34073412, 2009.

[14] C. Jennett, A. Cox, P. Cairns, S. Dhoparee, A. Epps, and T. Tijs. Measuring and defining the experience of immersion in games. International Journal of Human-Computer Studies,66(9):641-666, 2008.

[15] J. J. Kellaris and R. J. Kent. The influence of music on consumers' temporal perceptions: does time fly when you're having fun? J. of Consumer Psychology, 1(4):365-376, 1992.

[16] S. Luthman, T. Bliesener, and F. Staude-M"uller. The effect of computer gaming on subsequent time perception. CyberPsychology,3(1), 2009.

[17] R. E. Ornstein. On the experience of time. Penguin, Harmondsworth UK, 1969.

[18] P.-L. P. Rau, S.-Y. Peng, and C.-C. Yang. Time distortion for expert and novice online game players. CyberPsychology and Behavior,9(4):396-403,2006. [19] M. Slater, M. Usoh, and A. Steed. Depth of presence in virtual environments. Presence,3(2):130-140, 1994.

[20] P. Sweetser and P. Wyeth. Gameflow: a model for evaluating player enjoyment in games. ACM Computers in Entertainment,3(3), 2005.

[21] S. Tobin, N. Bisson, and S. Grondin. An ecological approach to prospective and retrospective timing of long durations: a study involving gamers. PLOSOne, 5(2), 2010. online, open access.

[22] J. Waterworth and E. Waterworth. Being and time: judged presence and duration as a function of media form. Presence,12(5):495-511, 2003.

[23] Z. Whalen. Play along - an approach to videogame music. Game Studies,4(1), 2004. online. [24] R. T. Wood, M. D. Griffiths, and A. Parke. Experiences of time loss among videogame players: an empiricalstudy. CyberPsychology and Behaviour, 10(1):38-44, 2007.

[25] D. Zakay and R. A. Block. New perspective on prospective time estimation. In V. D. Keyser, G. d'Ydewalle, and A. Vandieredonck, editors, Time and the dynamic control of behavior, pages 129-141. Hogrefe and Huber Publishers, Ashland, OH, 1998. [26] D. Zakay and E. Fallach. Immediate and remote time estimation: A comparison. Acta psychologica, 57:69-81, 1984. 